\title{
Etude de la croissance en hauteur du Cèdre (Cedrus atlantica Manetti) Utilisation d'un appareillage de mesure automatique
}

\author{
G. AUSSENAC, A. GRANIER, P. GROSS \\ I.N.R.A., Station de Sylviculture et de Production \\ Centre de Recherches forestières de Nancy, \\ Champenoux, F 54280 Seichamps
}

\section{Résumé}

La croissance du cèdre a été étudiée à l'aide d'un appareillage automatique permettant une mesure continue. Une description détaillée de l'appareil utilisé est donnée par les auteurs.

Les mesures effectuées ont montré que la croissance en hauteur du cèdre commence à Nancy à la fin du mois d'avril et se termine à la fin du mois de septembre.

La croissance se produit d'une façon générale pendant la nuit, mais lorsque le temps est couvert et pluvieux la croissance peut se poursuivre pendant la journée.

A partir de mesures effectuées les auteurs ont calculé le seuil apparent de végétation (température moyenne journalière) qui est égal à $6,6^{\prime \prime} \mathrm{C}$. Les auteurs montrent aussi que la durée de la période journalière de croissance est d'autant plus longue que l'état hydrique de l'atmosphère est élevé et que le rayonnement global est faible.

Les auteurs interprètent la croissance journalière en hauteur de la façon suivante :

- dans le courant de la nuit il y a croissance, en même temps l'arbre se réhydrate, ce qui se traduit par une augmentation de la turgescence;

- le matin la croissance ralentit alors que l'arbre est bien réhydraté ;

- dans la journée l'arbre voit son déficit hydrique augmenter. La rétraction de la pousse qui en résulte peut alors masquer la croissance en longueur au sens strict; déshydration.

- en fin de journée la croissance reprend et compense la rétraction due à la

\section{1. - Introduction}

La croissance de l'arbre peut être interprétée comme le résultat de l'interaction entre le génotype et les conditions écologiques, et parmi elles les facteurs climatiques et microclimatiques. Dans le cadre d'une étude sur le comportement écophysiologique du Cèdre (Cedrus atlantica Manet.), des recherches ont été entreprises pour preciser l'influence des facteurs climatiques sur la croissance en hauteur de cette essence. Dans ce domaine, très peu de données sont disponibles sur cette espèce fréquemment utilisée pour les reboisements en région méditerranéenne. 
L'étude de la croissance en hauteur nécessite des mesures périodiques. En conditions naturelles, les mesures sont le plus souvent faites à l'échelle de la semaine ou de la journée (Aussenac, 1975). L'auxanomètre, qui permet d'effectuer des mesures à des échelles de temps inférieures à l'heure, n'est utilisable qu'au laboratoire.

Nous rapportons ici les premiers résultats obtenus à partir de mesures (1) continues effectuées à l'aide d'un appareillage particulier, installé en conditions naturelles pendant une saison de végétation. Ces résultats sont complétés par trois années de mesures hebdomadaires.

\section{2. - Matériel et méthodes}

\subsection{Dispositif expérimental}

L'étude a été réalisée dans l'Arboretum d'Amance près de Nancy (altitude $250 \mathrm{~m}$ ), situé sur un sol brun lessivé qui s'est développé sur des marnes liasiques couvertes de limons. Nous donnons brièvement sur le tableau 1 un certain nombre de caractéristiques climatiques de cette station.

L'expérimentation a été réalisée dans un petit peuplement utilisé pour des études écophysiologiques. Nous avons suivi le débourrement et la croissance des arbres de ce placeau de 1977 à 1979 ; des mesures de potentiel hydrique ont été faites en 1976 et 1977. Les mesures de croissance en continu ont été effectuées sur un arbre dont nous donnons les principales caractéristiques ci-dessous :

\begin{tabular}{l|c|c|c}
\hline & Age & $\begin{array}{c}\text { Hauteur } \\
(\mathrm{m})\end{array}$ & $\begin{array}{c}\text { Diamètre à } 1,30 \mathrm{~m} \\
(\mathrm{~cm})\end{array}$ \\
\hline $\begin{array}{l}\text { Codrus atlantica } \ldots \ldots \ldots \ldots \ldots \ldots \ldots \ldots \\
\text { provenance Maroc }\end{array}$ & 16 ans & 4,80 & 11,4 \\
\hline
\end{tabular}

\subsection{Méthode de mesure}

Les premiers essais de mesure automatique ont commencé en 1977, il se sont poursuivis en 1978. En 1979 nous avons pu effectuer des mesures tout au long de la saison de végétation.

L'appareillage qui a été réalisé pour effectuer des mesures devait répondre à un certain nombre de spécifications, en particulier :

- possibilité d'une utilisation en conditions naturelles;

- bonne fiabilité.

(1) Etude en partie financée par la D.G.R.S.T. 
CROISSANCE DU CÈDRE

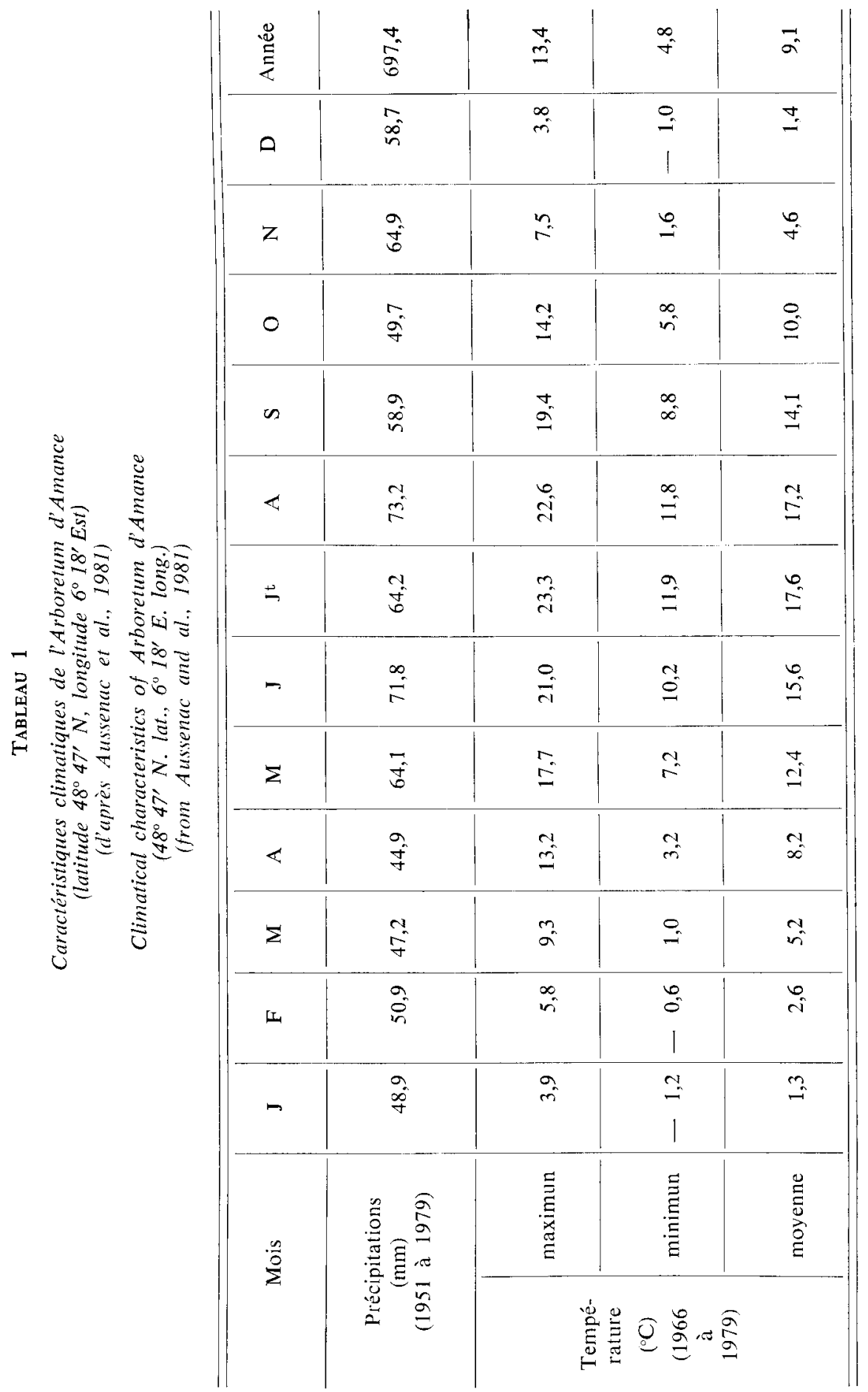


Principe de fonctionnement (figure 1)

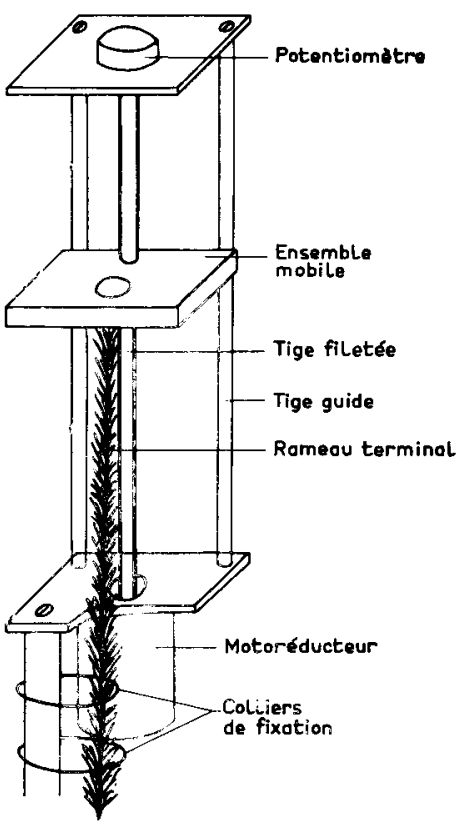

A - Schéma mécanique

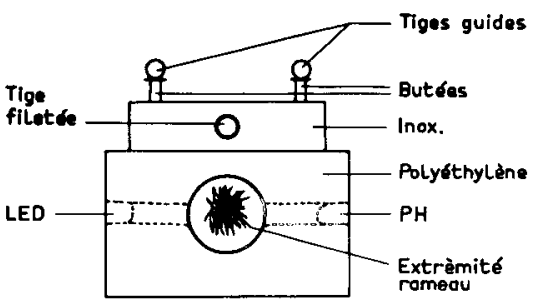

La LED ot le PH sont noyés dons de lo résine B - Détail de l'ensemble

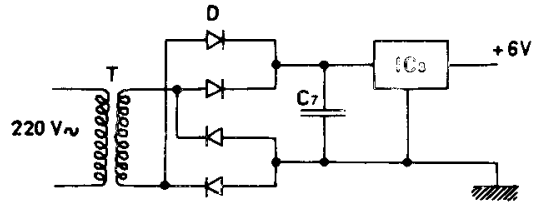

T Transformateur 220-9V 5 VA

D IN 4006 C, $220 \mu \mathrm{F} 20 \mathrm{~V}$

IC $C_{3}$ LM 7806

D - Alimentation $+6 \mathrm{~V}$

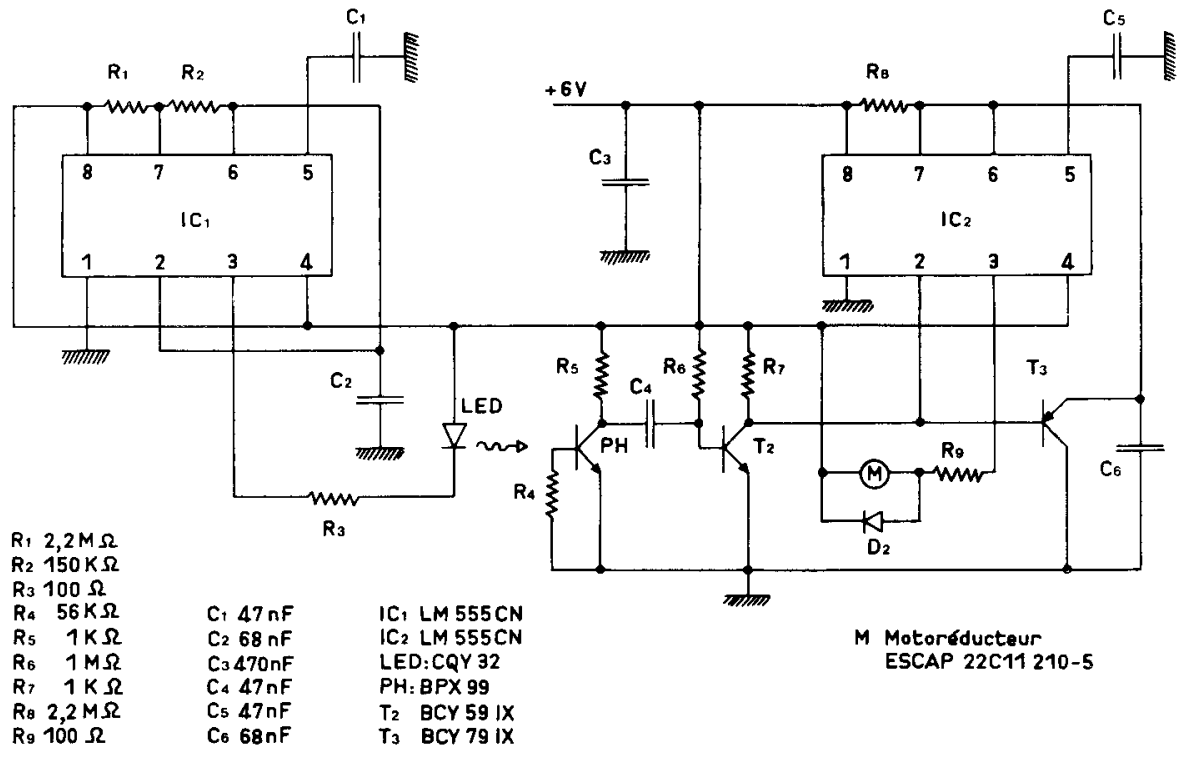

C - Schéma de l'ensemble électronique

FIG. 1

Schéma de l'appareillage de mesure de la croissance en hauteur
A et $B$ partie mécanique
$\mathrm{C}$ et $\mathrm{D}$ partie électronique

Diagram of the height-growth measuring equipment
$A$ and $B$ mechanical part
$\mathrm{C}$ and $\mathrm{D}$ electronical part 
Une diode électroluminescente et un phototransistor fixés sur un ensemble mobile forment une «barrière » infrarouge; le franchissement de cette barrière par la pousse provoque l'alimentation d'un moteur qui entraîne le déplacement de l'ensemble mobile jusqu'à ce que la barrière revienne juste au-dessus de la pousse. Un potentiomètre couplé à l'ensemble permet l'enregistrement.

\section{Fonctionnement de la partie électronique (figure 1)}

La diode électroluminescente LED fonctionnant dans l'infrarouge est alimentée en impulsions de durée $7 \mathrm{~ms}$ et de période $118 \mathrm{~ms}$ par le circuit $\mathrm{Ic}_{1}$ monté en oscillateur astable; le courant dans la LED est fixé à $50 \mathrm{~mA}$ par la résistance $\mathrm{R}_{3}$.

Le phototransistor $\mathrm{PH}$ (en fait un photodarlington) a sa sensibilité diminuée par la résistance $\mathbf{R}_{4}$ pour empêcher son point de repos d'approcher la région de saturation même par fort rayonnement solaire. Le condensateur $C_{4}$ transmet les variations de tension de collecteur du phototransistor $\mathrm{PH}$ au transistor $\mathrm{T}_{2}$ qui a son point de repos fixé à $5 \mathrm{~V}$.

Lorsque la pousse franchit la barrière infrarouge, la tension de collecteur du phototransistor $\mathrm{PH}$ ne varie plus, celle du transistor $\mathrm{T}_{2}$ reste donc à $5 \mathrm{~V}$; le condensateur $C_{6}$ qui se charge à travers la résistance $R_{8}$ peut atteindre la tension de basculement $(4 \mathrm{~V}) \mathrm{du}$ monostable construit autour du circuit $\mathrm{Ic}_{2}$. La sortie du monostable passe alors à l'état bas et alimente le motoréducteur $\mathbf{M}$ par l'intermédiaire de la résistance $R_{9}$ (cette résistance sert à limiter l'échauffement du moteur en cas de blocage mécanique).

Quand la barrière infrarouge de l'ensemble mobile entraîné par le motoréducteur $\mathbf{M}$ arrive juste au-dessus de la pousse, le phototransistor reçoit les impulsions infrarouges qu'il transforme en impulsions électriques envoyées sur le transistor $T_{2}$ via le condensateur $\mathrm{C}_{4}$; chaque front négatif bloque $\mathrm{T}_{2}$ alors que chaque front positif le sature amenant ainsi le potentiel de l'entrée TRIGGER du monostable à une valeur inférieure à $2 \mathrm{~V}$ qui permet son déclenchement : la sortie du monostable passe à l'état haut; le motoréducteur cesse d'être alimenté, le condensateur $C_{6}$ qui était déchargé pendant l'état bas du monostable peut se recharger à travers $R_{8}$ sans que toutefois son potentiel puisse atteindre la tension de basculement $\mathrm{du}$ monostable (4V) puisque $118 \mathrm{~ms}$ après le début de la charge, une nouvelle impulsion sur $T_{2}$ amènera le transistor $T_{3}$ à saturation et déchargera $C_{6}$ qui se rechargera ensuite pour un nouveau cycle.

Le monostable reste ainsi à l'état haut (motoréducteur non alimenté) tant que la pousse ne coupe pas le faisceau infrarouge. Le potentiomètre $P$ est directement monté en bout de tige filetée afin d'avoir une meilleure précision : lorsque l'ensemble mobile subit une translation de $1,25 \mathrm{~mm}$ (pas de la tige filetée) le potentiomètre effectue une rotation. Nous utilisons en effet un modèle à angle mécanique infini (3435 BOURNS). La plage morte électrique est de $10^{\circ}$, soit une erreur de $35 \times 10^{-3} \mathrm{~mm}$ (pour le pas de la tige filetée considérée) dans cette zone morte. La linéarité du potentiomètre est de 0,5 p. 100 , ce qui correspond à une erreur de $6 \times 10^{-3} \mathrm{~mm}$.

L'ensemble mécanique est réalisé en acier inoxydable dont le coefficient de dilatation linéaire avoisine $17 \times 10^{-6}{ }^{\circ} \mathrm{C}^{-1}$ : pour une hauteur moyenne de l'ensemble mobile de $200 \mathrm{~mm}$ et pour une variation de température de $10^{\circ} \mathrm{C}$ l'erreur absolue est donc de $34 \times 10^{-3}$, ce qui donne $40 \times 10^{-3} \mathrm{~mm}$ d'erreur absolue totale dans les conditions d'utilisation courante. 


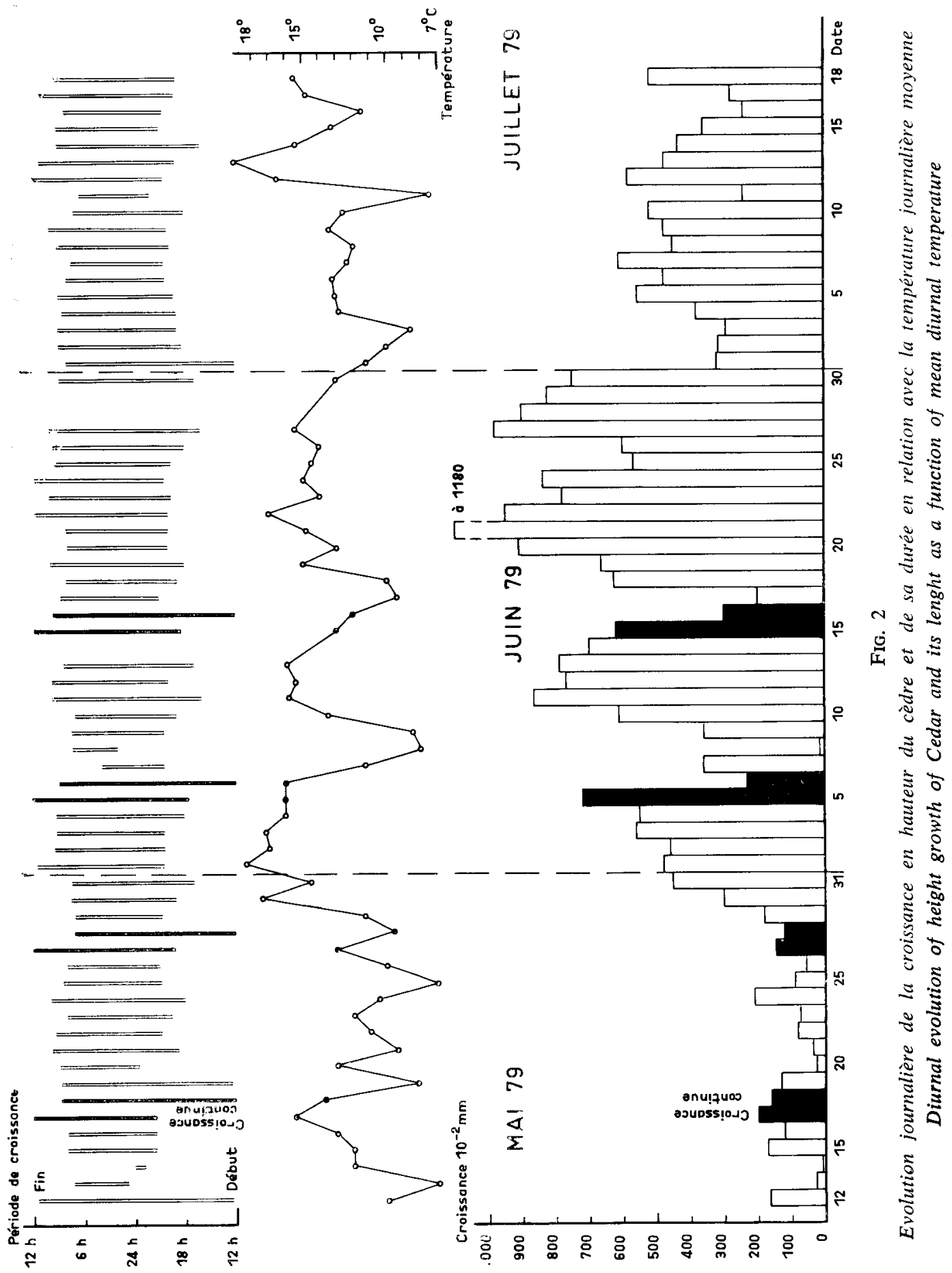




\section{3. - Résultats}

\subsection{Evolution journalière et saisonnière de la croissance}

La figure 2 représente pour la période du 11/05 au 20/07/79 l'évolution journalière de la croissance en hauteur. On note un maximum de croissance du 20 au 21 juin. Le débourrement, mesuré par la date du stade phénologique $b_{s, 5}$ (Aussenac, 1975) a eu lieu le 7/05/79, et la date d'arrêt de la croissance le 30/09/79. Des mesures effectuées les années précédentes indiquent d'une part que le débourrement de cette espèce a lieu en moyenne à la fin du mois d'avril et que d'autre part larrêt de la croissance se situe en moyenne dans la dernière quinzaine du mois de septembre.

\begin{tabular}{c|c|c|c}
\hline Année & $\begin{array}{c}\text { Date de } \\
\text { débourrement }\end{array}$ & $\begin{array}{c}\text { Date d'arrêt } \\
\text { de la croissance* }\end{array}$ & $\begin{array}{c}\text { Durée } \\
\text { de la croissance } \\
\text { (jours) }\end{array}$ \\
\hline 1977 & $24 / 4$ & $21 / 9$ & 150 \\
1978 & $26 / 4$ & $3 / 10$ & 160 \\
1979 & $7 / 5$ & $13 / 9$ & 129 \\
\hline
\end{tabular}

(\%) Date correspondant aux $98 \%$ de la pousse totale.

Dans les conditions climatiques d'Amance (AUSSENAC et al., 1981, tableau 1), le cèdre a une période de croissance longue et si on la compare à celle du douglas : 106 jours, du sapin pectiné : 55 jours, et de l'épicéa : 50 jours (Aussenac, 1975).

D'une façon générale, la croissance a lieu en phase nocturne (figure 3); cependant la durée de la croissance peut être plus ou moins longue, et dans certains cas elle peut se poursuivre sans arrêt pendant deux jours consécutifs. Les figures 4 et 5 donnent l'évolution de la croissance pour deux types de journées :

- temps couvert et pluvieux : figure 4 (humidité relative forte, rayonnement global faible). L'arbre a poussé de façon continue, mais sa croissance a été plus forte durant la nuit ;

- beau temps : figure 5 (humidité relative faible, rayonnement global fort). L'examen de cet exemple montre que la plus grande partie de la croissance de l'arbre s'est produite en période nocturne. Les seuils d'humidité relative et de rayonnement global correspondant au début et à l'arrêt de la croissance sont différents :

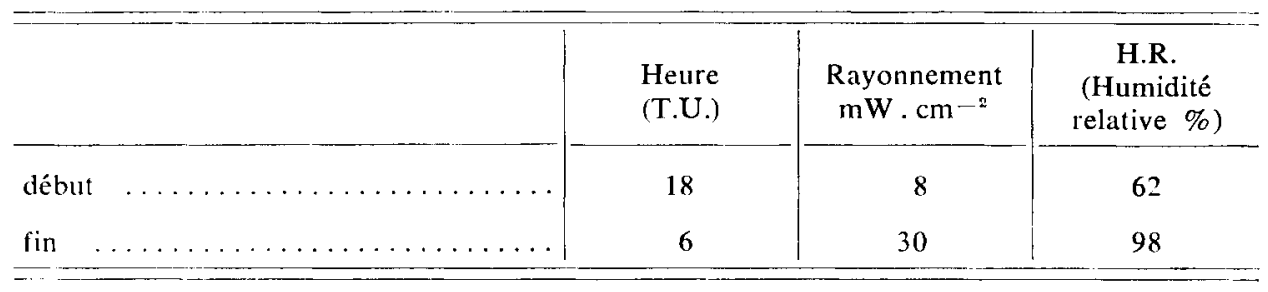




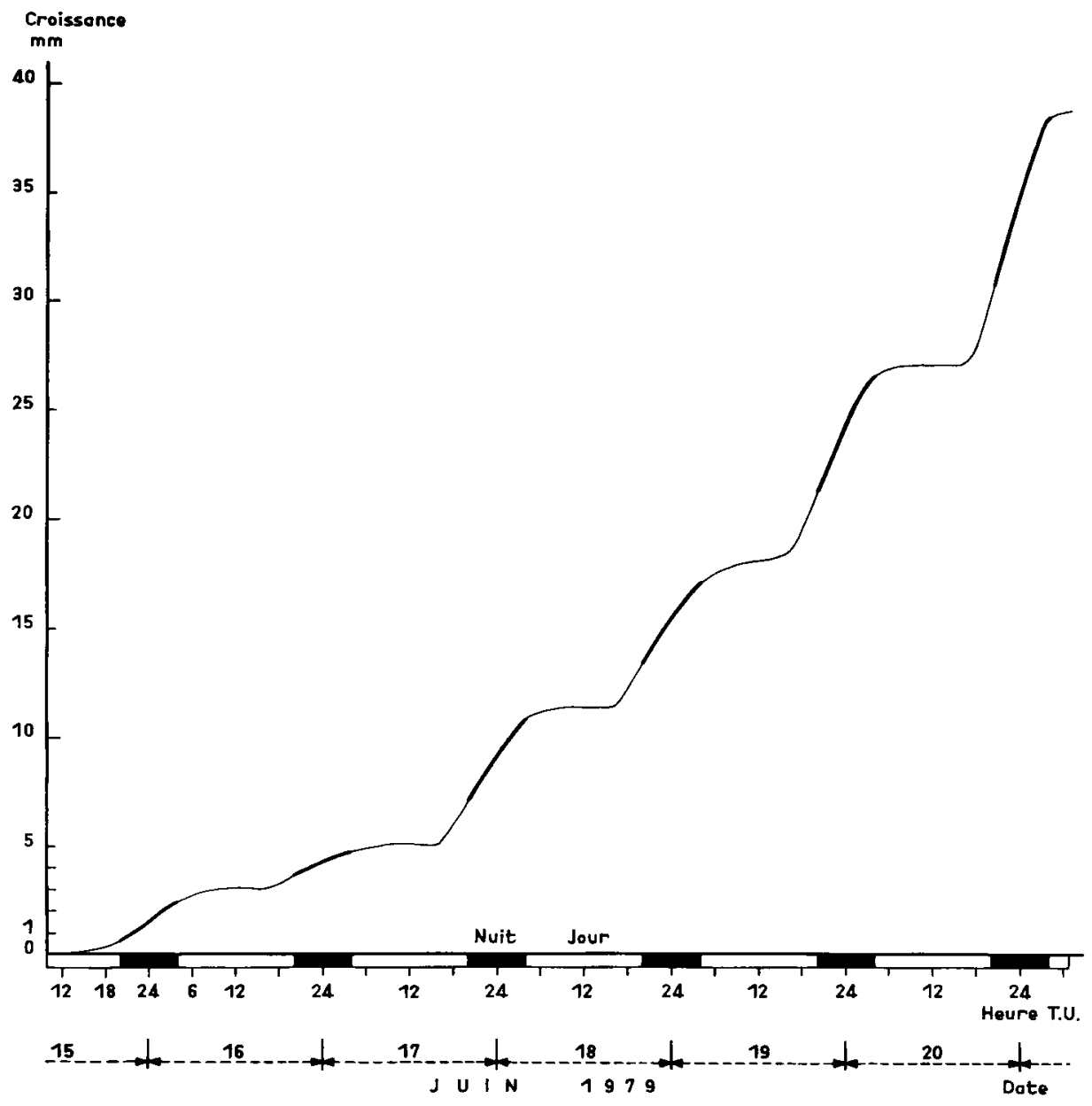

FIG. 3

Croissance en hauteur (cumulée) du cèdre du 15 au 20 juin 1979 Cumulative height growth of Cedar from June 15th to 20th, 1979 
Royonnement $\mathrm{mW} / \mathrm{cm}^{2}$

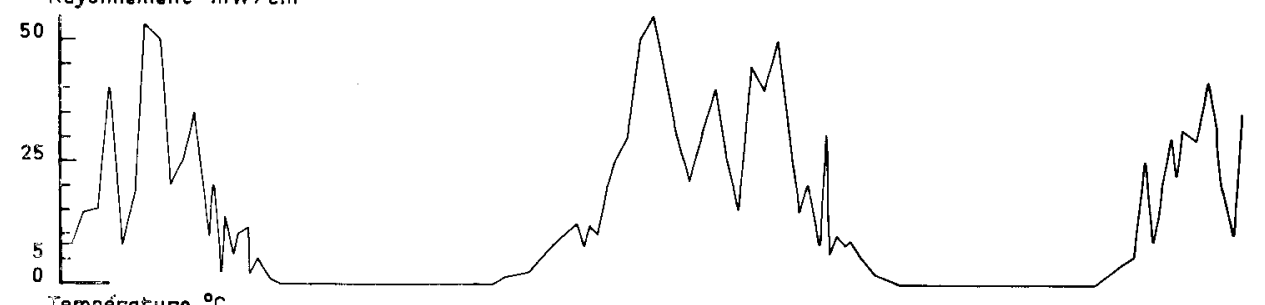

$49^{7}$ emperature ${ }^{\circ} \mathrm{C}$
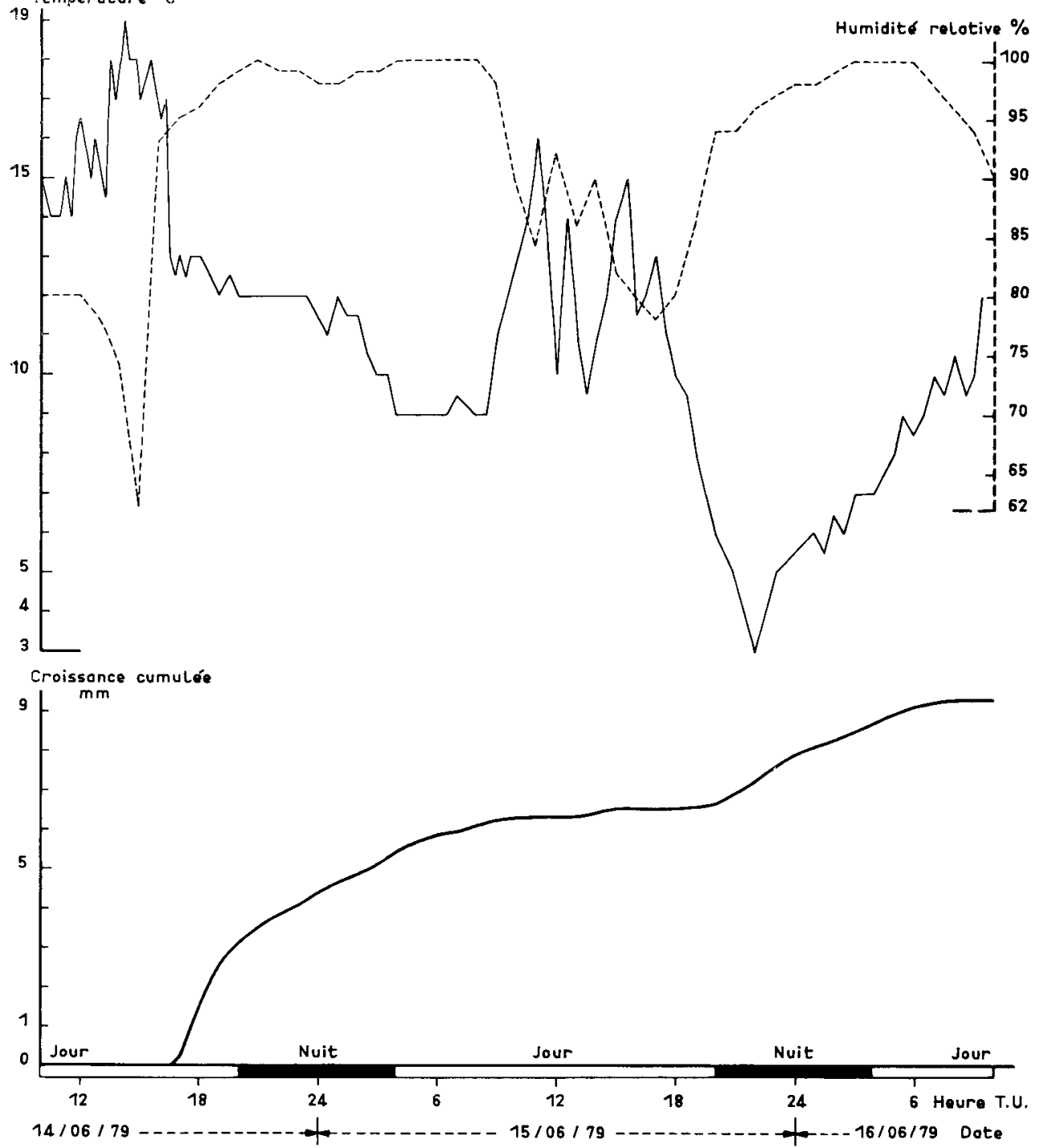

FIG. 4

Croissance en hauteur du cèdre par temps couvert et pluvieux, du 14 au 16 juin 1979 Height growth of Cedar in overcast sky and wet weather conditions, from June 14th to 16th, 1979 


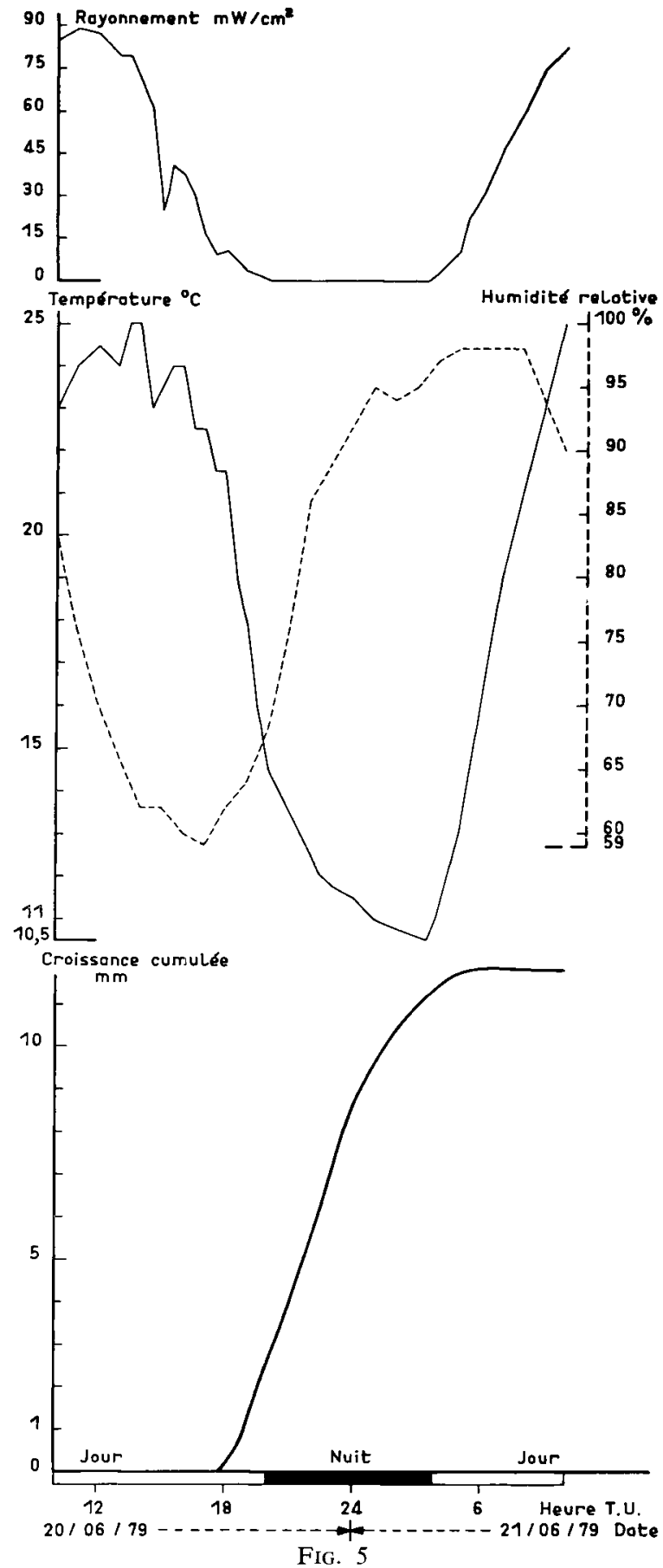

Croissance en hauteur du cèdre par beau temps les 20 et 21 juin 1979

Height growth of Cedar in sunny weather conditions on June 20th and 21st, 1979 


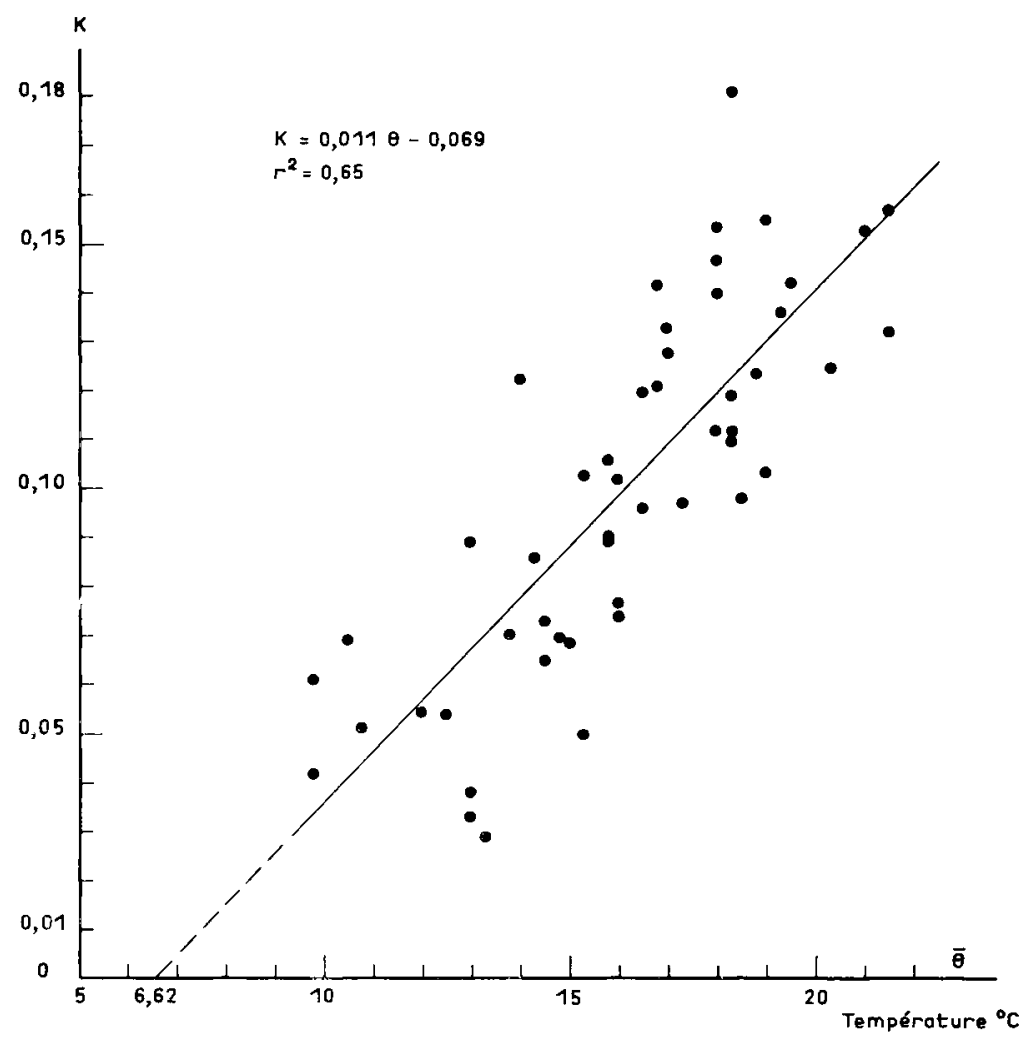

FIG. 6

Relation entre le facteur de croissance $K$ et la température moyenne journalière (de 12 heures à 12 heures)

Growth parameter $K$ as a function of mean daily temperature (from noon to noon)

\subsection{Relation entre la croissance et la température}

La figure 2 montre qu'il y a une relation entre l'accroissement journalier et la température moyenne pendant cette période de croissance. Nous avons représenté sur la figure 6 la relation entre la température moyenne journalière (de 12 heures à 12 heures) et le coefficient de croissance $K$ défini à partir de l'équation de ROBERTSON (1908) de la façon suivante :

$$
\mathbf{K}=\frac{1}{1\left(1-\frac{1}{\mathrm{~L}}\right)} \cdot \frac{\Delta \mathrm{l}}{\Delta \mathrm{t}}
$$

où 1 désigne la longueur de la pousse au temps $t$,

$L$ la longueur atteinte en fin de croissance,

$\Delta 1$ l'accroissement pendant une durée $\Delta t$. 
Il est alors possible de calculer la température pour laquelle $\mathrm{K}=0$; ce seuil est égal à $6,6^{\circ} \mathrm{C}$. Nous avons aussi étudié la relation entre $\mathrm{K}$ et la température moyenne de la période où il $\mathrm{y}$ a véritablement croissance, par exemple de 18 heures à 6 heures pour la période du 20 au 21 juin 1979. On obtient alors l'équation $\mathrm{K}=0,013 \theta-0.069$ avec $\mathrm{r}=0,76$.

Nous considérerons que le seuil de végétation apparent du cèdre est de $6,6{ }^{\circ} \mathrm{C}$, pour la température moyenne journalière.

\subsection{Relation avec le rayonnement global et l'humidité relative}

L'examen de l'évolution de la croissance chez le cèdre au cours de la saison de végétation indiquait (voir §3-1) qu'il y avait probablement une corrélation entre la durée de la croissance et certaines caractéristiques climatiques : rayonnement global et humidité de l'air. Une étude de corrélation multiple a fait apparaître la corrélation :

$$
\mathrm{T}=693,1-0,19 \mathrm{RG}+6,54 \mathrm{HR}\left(\mathrm{R}^{2}=0,45\right)
$$

avec $\mathrm{T}$ : durée de la période de croissance en minutes,

RG : rayonnement global en joules pour la période de 12 heures le jour $n-1$ à 12 heures le jour $\mathrm{n}$,

HR : humidité relative moyenne durant cette même période.

Il apparaît ainsi que la durée de la période de croissance (à l'échelle journalière) est corrélée négativement avec le rayonnement global et positivement avec l'humidité relative, ce qui indique que la durée de la période de croissance est d'autant plus longue que l'état hydrique de l'atmosphère est élevé.

\subsection{Etude des sommes de température}

Si l'on effectue la somme de température (au-dessus du seuil apparent de $6,6^{\circ} \mathrm{C}$, en degrés-jour), nous constatons que pour les trois années de mesure 1977 , 1978, 1979, la croissance en hauteur s'est terminée pour des sommes voisines de 1300 degrés-jour. Il faut remarquer que cette somme correspond en moyenne (à Amance) aux mois de mai, juin, juillet, août et septembre, c'est-à-dire à la totalité de la saison de végétation.

Il semble par ailleurs que chez le cèdre, il existe plusieurs poussées de croissance successives sans qu'il soit question ici de pousses différentes (comme les pousses d'août par exemple). C'est ce que nous pouvons constater sur la figure 7 qui donne l'évolution de la longueur en fonction de la somme de température.

\section{4. - Discussion et conclusion}

La croissance en hauteur du cèdre se produit d"une façon générale en phase nocturne. Nous avons vu que les seuils de début et d'arrêt de la croissance étaient différents tant pour le rayonnement global que pour l'humidité relative. Or, nous 


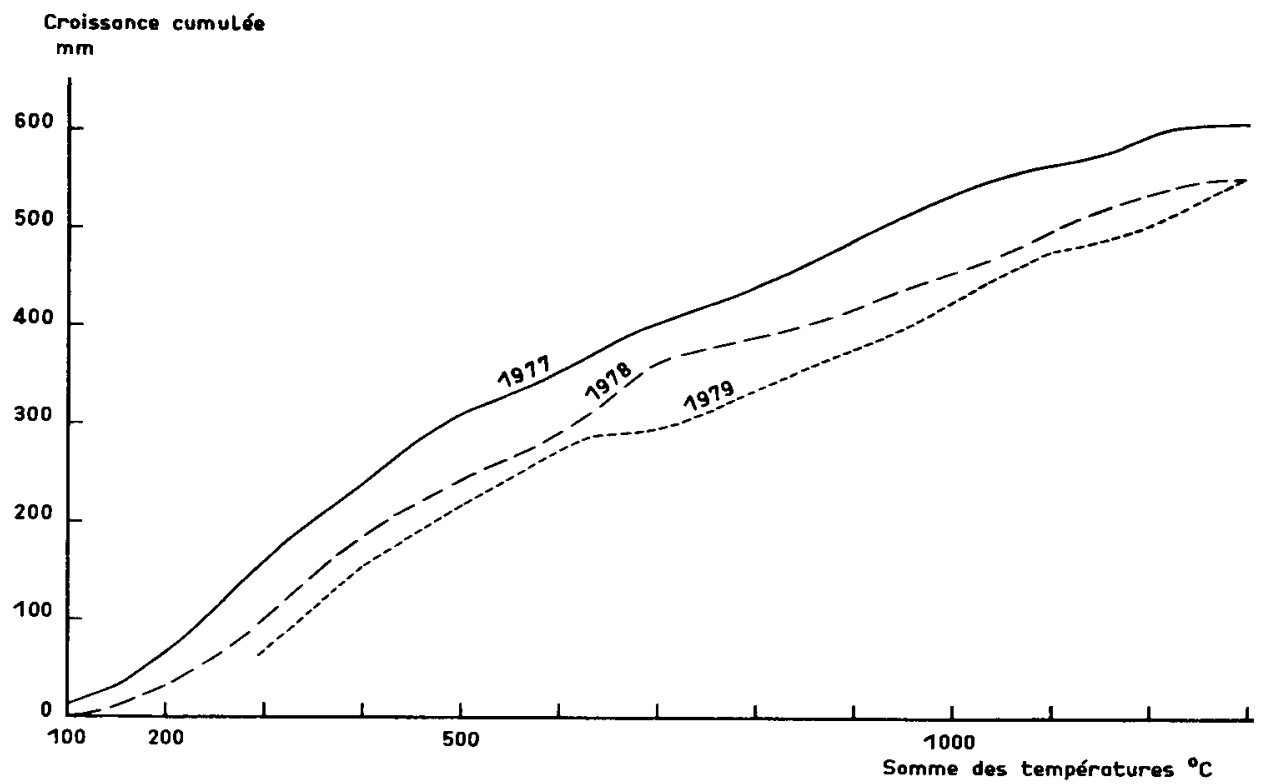

FIG. 7

Evolution de la croissance cumulée du cèdre en fonction des sommes de température au seuil $6,6^{\circ} \mathrm{C}$ pour trois années de mesure Cumulative height growth as a function of sum of temperature from the $6.6^{\circ} \mathrm{C}$ threshold for three years

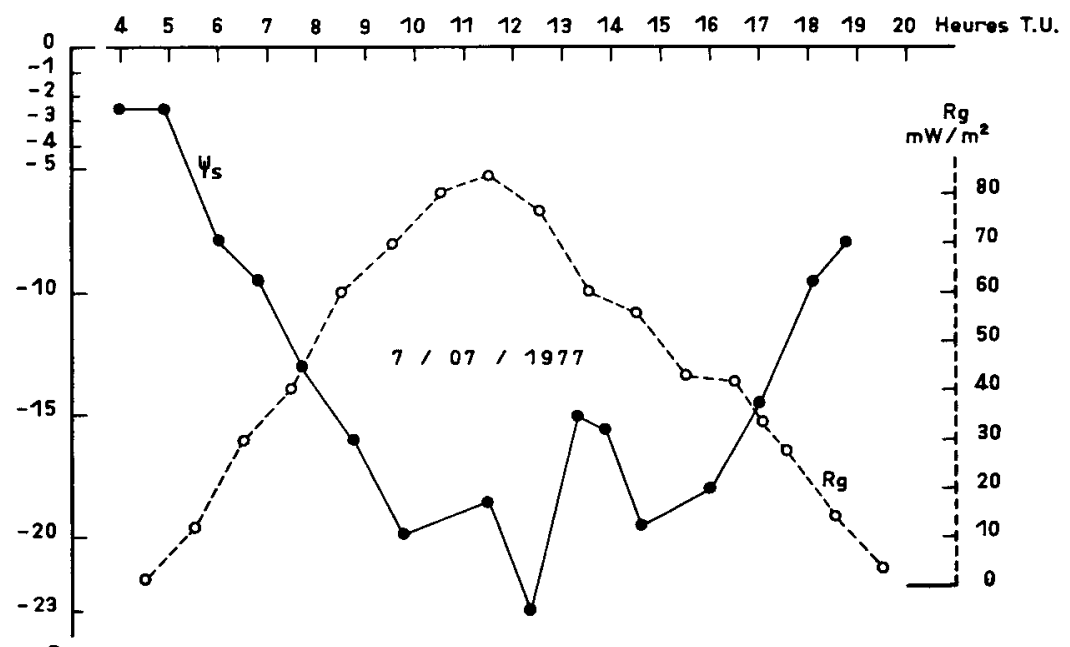

FIG. 8

Exemple d'évolution du potentiel hydrique ( $\Psi_{S}$ ) chez le cèdre en relation avec le rayonnement global $(R g)$, pour une belle journée Example of relationship between xylem water potential $(\Psi s)$ in Cedar and global radiation $(R g)$, in sunny weather conditions 
reportons sur la figure 8 l'évolution typique du potentiel hydrique (*) de rameaux de cèdres pour une belle journée lors d'une période de sécheresse moyenne. Le début de la croissance correspond à un potentiel de sève de - 10 bars et l'arrêt à $-2,5$ bars environ. On peut alors interpréter le phénomène de croissance de la façon suivante :

- dans le courant de la nuit il y a croissance en hauteur; dans le même temps l'arbre se réhydrate, ce qui se traduit par une augmentation de la turgescence;

- le matin, la croissance ralentit alors que l'arbre est bien réhydraté. Dans la journée, l'arbre voit ses échanges transpiratoires augmenter ce qui conduit à une déshydratation, donc à une rétraction de la pousse. FeElding, 1955 et Woorrall, 1973, ont pu observer ce phénomène de rétraction chez plusieurs essences.

Le fait que la croissance reprenne alors que l'arbre ne s'est pas entièrement réhydraté peut être interprété comme la résultante de deux phénomènes de nature différente : d'une part il y a rétraction dans le courant de la journée (par diminution de la turgescence), et d'autre part il y a croissance au sens strict du terme. En fin de journée cette croissance arrive à compenser la rétraction.

Plusieurs auteurs (KIENHOLz, 1934 pour plusieurs conifères, Worrall, 1973 pour l'épicéa) ont aussi montré que la croissance journalière était faible par rapport à la croissance nocturne. Lorsque la diminution de turgescence est faible (cas de la figure 4), la croissance est supérieure à la rétraction. Dans ce cas, la somme des deux phénomènes est positive et il y a alors une croissance mesurable. Nous avons schématisé l'évolution des différentes composantes de la longueur de la pousse sur la figure 9.

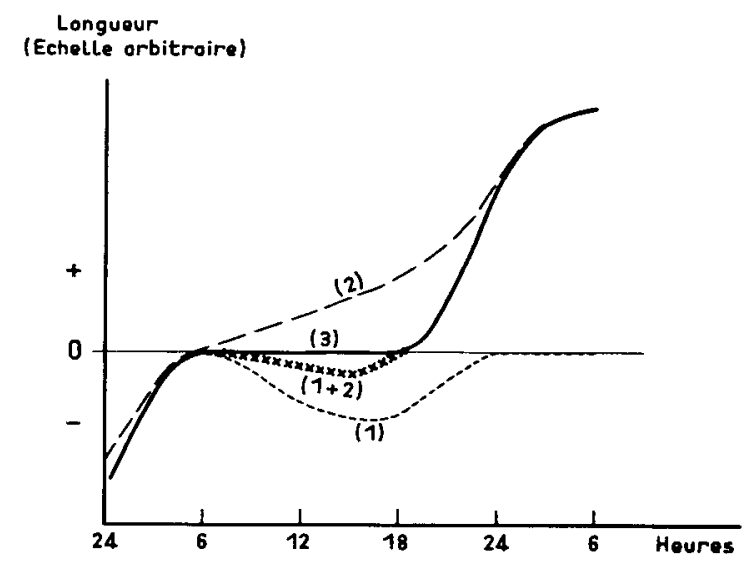

FIG. 9

Evolution schématique des différentes composantes de la variation en longueur de la pousse

(1) rétraction de la pousse

(2) croissance au sens strict

$(1+2)$ résultante de (1) et $(2)$

(3) accroissement mesuré

Schematic evolution of the various components of the lenght shoot variation

(1) shoot shrinkage

(2) actual growth

$(1+2)$ resultant of (1) and (2)

(3) measured growth

(*) Mesuré par la pression de sève suivant la méthode de la bombe de Schollander. 
Notre appareillage ne permet de mesurer que des variations positives de la longueur de la flèche; il n'est pas possible de suivre les rétractions de la pousse comme ont essayé de le faire Milne et al., 1977. Cette solution a été choisie pour simplifier le dépouillement des mesures.

Le seuil apparent de végétation trouvé pour le cèdre : $6,6^{\circ} \mathrm{C}$ (température moyenne journalière) doit être rapproché de celui trouvé pour d'autres espèces résineuses : Abies alba: $5,2{ }^{\circ} \mathrm{C}$, Abies nordmanniana : $6,2^{\circ} \mathrm{C}$, Picea abies : $4,1{ }^{\circ} \mathrm{C}$, Pseudotsuga menziesii : $5,8^{\circ} \mathrm{C}$ (AussenAC, 1975). Bien que montagnard dans son aire naturelle le cèdre apparaît ici comme plus thermophile que les espèces citées précédemment.

La croissance du cèdre peut être relativement longue si on la compare à celle d'autres résineux, le sapin par exemple. Certains auteurs ont admis l'idée que l'arrêt précoce de la croissance en hauteur était une adaptation pour résister à la sécheresse (Bouvarei, 1961). En ce qui concerne le cèdre il ne semble pas que cela soit le cas puisque la période de croissance peut aller de mai à fin octobre. Il est probable que dans les régions à sécheresse estivale marquée, la période de croissance puisse être réduite comme le laissent supposer certaines expérimentations en cours. On peut également penser que dans ces régions si les conditions d'alimentation en eau sont satisfaisantes, la croissance peut se prolonger au-delà des 1300 degrés jours que nous avons calculés ici. Des mesures de date d'arrêt de végétation effectuées dans le midi de la France (Pradal, 1979) rendent vraisemblable cette interprétation.

Reçu pour publication en août 1980.

\section{Summary \\ Study of height growth of Cedar (Cedrus atlantica Manetti). Use of an automatic equipment}

Height growth of Cedar has been studied by means of an automatic equipment allowing continuous recording. Its detailed description is given by the authors.

The measurements have shown that height growth of Cedar in Nancy starts at the end of April and stops at the end of September.

The growth generally occurs at night, but in overcast sky and wet weather conditions the growth may continue all day long.

From these measurements, an apparent vegetation threshold (mean daily temperature) has been calculated : it is equal to $6.6^{\circ} \mathrm{C}$. The authors have pointed out that the length of the daily growing period increases with atmospheric humidity and decreases with global radiation.

An explanation of daily growth phenomenon is then given :

- at night the terminal shoot grows, while the tree progressively gets rehydrated bringing about an increase of its turgescence;

- at sunrise the growth decreases as the tree is well hydrated;

- during the day, the water deficit of the tree increases. The shrinkage of the shoot exceeds its growth;

- at the end of the day, the shoot growth makes up for the shrinkage. 


\section{Références bibliographiques}

Aussenac G., 1975. Etude de la croissance en hauteur chez quelques résineux. Effet de la température. Ann. Sci. for., 32 (1), 1-16.

Aussenac G, 1975 Couverts forestiers et facteurs du climat, leurs interactions, conséquences écophysiologiques chez quelques résineux. Thèse ès Sciences, Nancy, $213 \mathrm{p}$.

Aussinac G., Desueunes J.M. et Willm F., 1981. Climatologie de l'Arboretum d'Amance. Rev. for. fr., XXXIII 1, 29-38.

Bouvarel P., 1961. Observations sur la date d'aoûtement de quelques provenances françaises d'épicéa. Ann. Ec. natl. Eaux Forêts Stn Rech. Exp. for., 18 (1), 99-129.

FIELDING J.M., 1955. The seasonal and daily elongation of the shoots of Monterey pine and the daily elongation of roots. Commonw. Aust. for. Timb. Bur., Leaflet $\mathrm{n}^{\circ} 75$.

Kienholz R., 1934. Leader, needle, cambial and root growth of certain conifer and their relationship. Bot. Gaz., 96, 73-92.

Milne R., Smith S.H. and Ford E.D., 1977. An automatic system for measuring shoot length in sitka Spruce and other plant species. J. appl. Ecol., 14, 523-529.

Pradal F., 1979. Variabilité génétique et écophysiologique du cèdre. Mémoire E.N.I.T.E.F., $89 \mathrm{p}$.

Robertson, 1908. Archiv Entwicklengmesh. Org., 25, 181.

Worrall J., 1973. Seasonal, daily and hourly growth of height and radius in Norway Spruce. Can J. for. Res., 3, 501-511. 